\title{
International Cooperation in Developing Countries: Reducing Fatalism and Promoting Self-Efficacy to Ensure Sustainable Cooperation
}

\author{
Daniela Pajardi ${ }^{1, * \mathbb{C}}$, Monia Vagni ${ }^{1}$, Viviana La Spada ${ }^{1}$ and Serena Cubico ${ }^{2, * \mathbb{C}}$ \\ 1 Department of Humanities, University of Urbino, 61029 Urbino, Italy; monia.vagni@uniurb.it (M.V.); \\ vivianalaspada@gmail.com (V.L.S.) \\ 2 Department of Business Administration, University of Verona, Verona 37129, Italy \\ * Correspondence: daniela.pajardi@uniurb.it (D.P.); serena.cubico@univr.it (S.C.); \\ Tel.: +39-072-2305814 (D.P.); +39-045-8028132 (S.C.)
}

Received: 18 December 2019; Accepted: 8 January 2020; Published: 10 January 2020

\begin{abstract}
International cooperation projects aim to support populations in developing countries or affected by emergency situations and to promote their wellbeing in a coherent way and in line with the 10th Sustainable Development Goal and with the principles of the psychology of sustainability and sustainable development. This study analyzed the ways in which such projects influence two psychosocial variables, fatalism and self-efficacy, which are of great importance in determining the attitude of people to promoting change and improving their living conditions by themselves. The sample $(N=510)$ consists of adult users of Caritas Italiana projects in developing countries, namely, 161 individuals in Argentina, 123 in Bosnia, 96 in Sierra Leone, and 130 in Sri Lanka. The results indicate that the very fact of being involved in cooperation projects, both economic welfare and social promotion projects, favors a reduction in fatalistic attitudes and that greater perception of self-efficacy predicts a reduction in fatalism. Specific effects are presented in relation to the different cultures, and education levels of the countries analyzed.
\end{abstract}

Keywords: fatalism; self-efficacy; social cooperation; sustainability

\section{Introduction}

Psychology of sustainability and sustainable development [1-3] constitutes a new research area in the field of sustainability science fostering its trans-disciplinary perspective. This new research area widens the concept of sustainability based on ecological and socio-economic environments. Overcoming the traditional framework based on the three "Es" (economy, equity, and ecology), the psychological perspective pays attention to individual characteristics and resources [4-6] and sustainable development [7] in the environment/s. As regards this framework, special attention should be given to the integration and harmonization among resources, sustainable development, and the surrounding socioenvironmental context [8].

This study is in line with the 2015 European Year for Development objectives, promoted by the European Commission in favor of sustainable development [9], and with the United Nations Objectives for Sustainable Development (United Nations, 2018, Objective 10: Points 10.2 and 10.3), since it aims to promote social inclusion and competences at the political level, of the community, and to reduce inequalities in populations. Therefore, in line with this objective, to reduce poverty and inequalities, it is necessary to focus on inclusive growth that involves all three dimensions of sustainable development: Economic, social, and environmental. Specifically, the United Nations Objective 10.2 aims to strengthen and promote the social inclusion of the entire population, regardless 
of gender, religion, economic status or race differences, whereas Objective 10.3 aims to provide equal opportunities and reduce inequalities, including through the promotion of specific social and political actions [10]. The present study considers psychological aspects to foster sustainable cooperation according to the new framework of the Psychology of Sustainability and Sustainable Development.

The present study considered international cooperation initiatives conducted by Caritas Italiana, an association created by the Italian Episcopal Conference, which has a leading role at the national and international levels in projects for the assistance and the social development in different countries worldwide. The types of international cooperation projects that Caritas Italiana implements are for both economic support and promotion and social inclusion. However, these are oriented to the so-called decentralized cooperation, which is aimed at developing local autonomies and which, 'responding to organized civil society, they apply a new concept of development, human development, based not only on economic indices or macro interventions, but on the direct participation of the recipient and mutual exchange between communities' [11].

The main variables considered in this study are fatalism [12-14] and self-efficacy $[15,16]$ in the context of sustainable primary prevention projects aimed at improving the quality of life [1] and at promoting social harmonization in different geographical and cultural contexts [17].

Fatalism and self-efficacy are variables that have in their theoretical construct the fact that they are influenced by different cultural, social, economic, health, education, and lifestyle conditions [12,18]. These characteristics make them among the most widely studied psycho-social constructs in different socio-cultural contexts.

Thus, this study presents a novel analysis of experiences of social cooperation in different countries in the social contexts of inequality and socioeconomic precariousness and aims to develop a little-explored research area on the correlation between these two constructs.

\subsection{Fatalism and Self-Efficacy: Psychosocial Variables and International Cooperation}

Fatalism is a factor that influences the possibility of acting on one's external and internal reality: The literature defines it as a set of beliefs that includes dimensions such as predetermination, pessimism and the attribution of life events to chance [14]. According to this construct, all events occur in an unavoidable way, and therefore, it causes attitudes of passivity with respect to reality that is perceived as inexorably flowing [19]. Alvarez et al. [20] show that users who receive support that is oriented towards favoring their autonomy then show greater empowerment and feel more competent than those who receive only material support, which is more oriented towards dependence. The authors argue that assistance projects, regardless of their purpose, must, above all, promote the autonomy and development of decision-making skills.

Fatalism has been studied in different social and organizational contexts, which demonstrates how it can represent an interesting key to understanding social reality. For instance, D'Orlando et al. [21] found that on the socioeconomic level, the psychological consequences of periods of jobless employment are more serious in more fatalistic subjects than in others, because the former believe they can do nothing to change their unemployment status. Different studies investigate fatalism from the point of view of clinical psychology, and these show that a fatalist attitude reduces the ability to adopt adaptive behaviors in the face of announced natural disasters. In addition, these studies report on the negative consequences of such disasters on psychophysical wellbeing. For example, fatalism amplifies the degree of post-traumatic suffering in the victims of the aforementioned disasters [22,23]. Some of these studies agree that fatalism is a complex cognitive-type construct composed of several variables. Some studies place specific attention on these variables, whereas others study its integration. For example, when we discuss fatalist subjects, we refer to those who prefer an external locus of control [24], but fatalism is also linked to the dimension of fate, luck, and predetermination of the discomfort or disease [25] and the concept of pessimism and negative expectations with respect to one's state of health [13]. 
Precisely in relation to health, a correlation has been studied with belonging to populations that are in a state of discomfort and social exclusion, thus highlighting how precarious socioeconomic or cultural conditions favor a greater level of fatalism and difficulty in accessing the forms of care and prevention [26-28]. Some authors argue that in disadvantaged populations that have a low socioeconomic status, fatalist beliefs can also be traced to a realistic assessment of their condition, in the face of concrete barriers that are difficult to overcome even with individual effort $[28,29]$. Further, educational level and economic level seem to be two variables that influence fatalism [30], which, according to Shen et al. [14] are 'the basis for interventions aimed at reducing fatalism and enhancing behavioral changes' (p. 9). In particular, with respect to the level of education, Ruiu [30] highlights that education allows one to be more aware of one's own abilities, and therefore less fatalistic, based on the World Values Survey data, a global survey on the values and beliefs of individuals in more than 80 countries, correlated with demographic information, economic conditions, political preferences, values, attitudes, and religions.

Awareness of one's abilities can be associated with the concept of self-efficacy. Bandura [15] defines it as the set of beliefs that people have about their ability to organize and direct their skills and resources to implement an action that will lead them to the desired goal. The extent to which an individual feels effective in undertaking a certain action is a basic psychological process, which leads to the development of a relational and social change and triggers an empowerment process. That is, this process leads to promotion not so much of technical knowledge as of new skills or already existing ones in individuals, allowing them to become actors in their own lives. The sense of self-efficacy influences different cognitive, affective, and behavioral factors and can help individuals tackle even stressful situations. Some scholars identify it as a more general operation, which concerns the perception of one's own ability to deal with a wide range of situations that are not only stressful but also new or demanding. In this case, we speak of general self-efficacy (GSE) [16,31,32]. Those with high levels of self-efficacy use more active coping strategies and humour. They are information-seeking individuals and have a more combative spirit in dealing with difficult situations. Conversely, those with low self-efficacy more frequently assume passive attitudes and manifest guilt feelings [32]. In addition, this construct has been extensively studied in the health field [33], and it has been highlighted as a determinant variable on the therapeutic success [34]. Further, high self-efficacy mediates the negative effects of daily stress on mental health [35].

\subsection{Fatalism and Self-Efficacy: Theoretical and Application Links}

Fatalism and self-efficacy are two constructs that have been widely studied independently of each other, and, in different cultural and application contexts, some studies have theoretically identified and empirically demonstrated the correlation [14,36,37], as both of them involve causal attributions, beliefs, and empowerment and investigate the manner in which people's attitudes and behaviors can change in different situations.

In this regard, the choice of these variables was suggested by the theoretical constructs of the variables themselves, describing them as two somewhat antithetical attitudes of the individual towards different situations of their personal life and the possibility of actively operating with respect to the possibilities of change, development, and access to a better condition of life [36,37]. Fatalism represents an attitude of passivity towards the possibility of changing a situation, whereas self-efficacy represents a proactive attitude.

The present study investigates both the relationship between these two constructs and the clash between these in a cross-cultural context. Shen et al. [14] link self-efficacy and fatalism, by analyzing data on a sample consisting of individuals of Hispanic and African American ethnicities who had different levels of education, and emphasize that the dimensions of lack of control and predetermination of events, typical of fatalist subjects, are indicators of low self-efficacy. Moreover, Taylor and Wilson [37] find this correlation for a sample of university students. One study [36], which is related to the present study in terms of the condition of the examined sample, considers fatalism an individual attitude that 
facilitates the lack of expectations of success in improving living conditions. Therefore, such individuals have a low level of self-efficacy, particularly in situations of economic and cultural poverty, such as those of the sample of individuals from Ethiopia that the study [36] examined. The authors [36] relate fatalism to the locus of control and self-efficacy, and they highlight fatalistic beliefs as indicators of a small aspiration gap and low self-efficacy. Go and You [38] show that the search for online information on cancer, even if it fails to diminish fatalistic attitudes, increases self-efficacy, promoting preventive behaviors with respect to cancer.

Bandura emphasizes that self-efficacy is a universally recognized, basic construct, whose values can change both for aspects of specific cultures and for the influence of psychosocial variables and socioeconomic conditions [18]. In particular, collectivist-oriented cultures show less consideration for the role of self-efficacy than do individualistic-oriented cultures: Scholz et al. [39] attribute the reason for this difference to the greater value given by collectivist cultures to the actual effort and commitment (hard work and effort) with respect to a transversal ability such as self-efficacy.

Luszczynska et al. [31], who conducted a study on inhabitants of 'emerging and developing economies' [40], including Costa Rica, Poland, and Turkey, and on inhabitants of 'advanced economies', including Germany and the United States, highlight similar cross-cultural patterns in the positive correlation between GSE and psychological constructs such as optimism, self-regulation, self-esteem, and academic performance, and in the negative correlation between GSE and anxiety and depression.

Self-efficacy has been studied in relation to educational level since these are conceptually connected: the possibility of having successful experiences increases the levels of self-esteem and self-efficacy [15]. The earliest studies on self-efficacy show that the level of school education influences self-efficacy. On a conceptual level, self-efficacy and the level of education have a positive correlation, which is interpreted as a recognition that a goal must be achieved, and this correlation serves to strengthen self-esteem. The level of education does not have an absolute or linear correlation with self-efficacy, but it is one of the variables that affect self-efficacy and that must be read in an integrated way with the other social variables, some of which may be expectations and style of educational system for parents, and the educational style of teachers, peer feedback [41].

We have chosen to use the GSE Scale because it presents several national validations, and therefore, it is a versatile tool to be administered to populations of different ethnicities and cultures [31,32,39].

\subsection{Aims of the Study}

This study involved the administration of the identified tools to users who use the intervention of Caritas Italiana and interact with them directly or through the mediation of local operators and Italian volunteers present on site. The objective was to identify the influence of two different types of cooperation (one of a purely economic assistance nature and the other of social promotion) on the fatalistic attitude of the users, through a test-retest at the beginning and end of the project. The self-efficacy level was also measured to detect its association with the fatalistic attitude [14,36-38].

This study aims to identify psychosocial variables that can be taken into account in planning international cooperation interventions, and that can promote attitudes aimed at improving not only the individual's own personal wellbeing and quality of life but also social, political, and economic self-determination.

Thus, this study considers an ethical-moral perspective of promoting local autonomy and independence as well as the sustainability of the assistance and autonomy of the local population with respect to external aid. Hence, the study's goals are in line with the aforementioned goals of the United Nations and with the approach of the psychology of sustainability [3], which involves both assistance and sustainable development.

\subsection{Hypothesis}

In the present study, the following hypotheses were investigated: 
- Hypothesis 1: Social cooperation interventions promote a greater sense of self-efficacy in different populations because these stimulate positive attitudes and experiences among individuals and the possibility of changing their condition [1].

- Hypothesis 2: The level of fatalism varies over time, following a cooperation intervention and depending on the country of origin.

\section{Materials and Methods}

\subsection{Study Context}

This study considered four countries, which have different types of intervention projects that concern the populations of all countries:

The projects for each country were economic support (welfare project) and social promotion projects (meetings to support inclusion, a sense of community, and individual and group empowerment). The study context is as follows:

1. South America (Argentina)

2. Africa (Sierra Leone)

3. Asia (Sri Lanka)

4. Europe (Bosnia)

\subsection{Participants}

The present study involved 510 participants from four different developing countries [40] to whom the interventions offered by Caritas Italiana were addressed. In particular, the participants had received interventions as part of one of the two types of assistance projects considered in this study. One project involved only economic or material support to the populations (e.g., delivery of food packages and family allowances to the participants), and the other was a social promotion project/intervention that aimed to encourage a greater level of socialization, cultural integration, and aggregation (e.g., campaigns to promote women's autonomy, the right to vote and projects to develop social participation in small communities).

The intervention lasted 12 months and the interested populations were met regularly by Caritas Italiana volunteers. The reference sample consisted of a total of 510 individuals from the four countries considered in the present study:

1. Argentina $=161$ participants comprising $31.6 \%$ of the sample, average age of 34.04 years ( $\mathrm{SD}=12.41), 23$ males $(14.2 \%)$ and 138 females $(37.9 \%)$, average years of study 7.67 (SD = 3.43);

2. Bosnia $=123$ participants comprising $24.1 \%$ of the sample, average age of 33.85 years $(\mathrm{SD}=11.30)$, 56 males $(45.5 \%)$ and 67 females $(54.5 \%)$, average years of study $13.27(\mathrm{SD}=2.23)$;

3. Sierra Leone $=96$ participants comprising $18.8 \%$ of the sample, average age of 39.97 years $(\mathrm{SD}=11.16), 67$ males $(69.8 \%)$ and 29 females $(30.2 \%)$, average years of study $14.57(\mathrm{SD}=2.47)$;

4. Sri Lanka $=130$ participants comprising $25.5 \%$ of the sample, average age of 38.50 years $(\mathrm{SD}=14.90), 26$ males $(20 \%)$ and 104 females $(80 \%)$, average years of study $6.74(\mathrm{SD}=3.24)$.

All participants were asked to complete the fatalism questionnaire at their first meeting to join the project [14]. At the last meeting, which was held after 12 months, the participants were again asked to complete this questionnaire (R_Fatalism), to verify whether some cultural beliefs that inhibit proactive behaviour were reduced, in favor of greater possibilities of the implementation of individual resources, trust, and decision-making autonomy. The last three variables are described as relevant in the psychology of the sustainability field [2]. Since the school systems were different and especially given that some participants, due to economic and social difficulties, suffered an interruption in their studies, the "years of study" variable was considered in the study rather than the qualification obtained. 
Fatalism and self-efficacy are attitudes that are strongly rooted in a person, and so it is obvious that a long period of time is necessary to modify them. The time span considered was 12 months in consideration of the duration of the project, which is quite a long period of time.

The last three variables are described as relevant in the psychology of the sustainability field [2]. Participants were also administered the GSE scale [16] to detect whether any changes in fatalistic conceptions are related to the general sense of self-efficacy.

A local cultural mediator, who was a local Caritas Italiana official, and the contact person for our project, translated the inventory. They had the required project experience and had acquired knowledge of the target population over a long period. Thus, these people were able to offer not only linguistic but also cultural adaptations of the instruments. Before administering the translated instruments, a pilot study was conducted to verify the effective understanding of the items.

All participants presented the ability to read and answer questions from the questionnaires independently. During the administration of the tools, the local cultural mediator was present and provided clarifications on the questions, where they were requested.

\subsection{Instruments}

\subsubsection{Fatalism Scale by Shen, Condit and Wright}

The Fatalism Scale [14] is composed of 20 items. The original scale developed by Powe (Fatalism Inventory Scale) in 1995 consists of 15 items that investigate four components of fatalism: Fear, predetermination, pessimism, and the inevitability of death. In the version we used instead, in which some items on predetermination and pessimism have been adapted and others added, it is investigated on three dimensions that, according to the most recent studies, constitute the construct of fatalism: Predetermination, luck, and pessimism. Data from a web-based national survey showed that the scale was unidimensional, and it showed good reliability (alpha $=0.88)$ [14]. A typical item that investigates predetermination is 'How long I live is predetermined'. A typical item of the luck dimension is 'How long I live is a matter of luck'. Finally, a typical item of the pessimism dimension is 'I will suffer a lot from bad health'. The response modalities of the Shen et al. scale [14] range from 1 to 6 ('true', 'almost always true', 'often true', 'sometimes true', 'rarely true', and 'false'). However, in the pre-test phase, we found that participants from certain populations found it difficult to accurately distinguish between the differences in responses on a Likert scale articulated on so many levels. Therefore, in the second round of the test-retest phase we decided to reduce the responses from 6 to 3 levels $(1=$ 'true', $2=$ 'quite true', 3 = 'false'). This pre-test was also conducted after a linguistic and cultural comparison with local volunteers and operators for standardizing the content of the questions and the response modalities for all the countries involved. Therefore, the total scores range from 20 to 60 .

\subsubsection{General Self-Efficacy Scale of Schwarzer and Jerusalem}

Schwarzer and Jerusalem's General Self-Efficacy Scale [16] is composed of 10 items that have a four-step Likert response, namely, 1 (not at all true), 2 (hardly true), 3 (moderately true) and 4 (exactly true). The total scores range from 10 to 40 . The GSE is composed of 10 items. Some typical items are: "I can always manage to solve difficult problems if I try hard enough", "When I am confronted with a problem, I can usually find several solutions", "If I am in trouble, I can usually think of a solution", "Thanks to my resourcefulness, I can handle unforeseen situations".

The literature has a version of the scale in Spanish, which was used for the participants of Argentina (Cronbach's alpha: 0.90). For other countries, the English version (which in different Anglo-Saxon countries has Cronbach 'alphas between 0.76 and 0.90) was used as the basis for local operators in Bosnia, Sierra Leone, and Sri Lanka and then translated into their respective local languages. Given the limited extent of the participants in each country, it was not possible to calculate Cronbach'alpha value. 


\subsection{Data Analysis}

Some preliminary calculations were performed to detect the level of correlation and the average differences between the main variables examined in the present study. Subsequently, an analysis of univariate variance (ANCOVA) was performed to detect the effects of the study variables Country Group and Project (assistance vs. social promotion) on self-efficacy. Lastly, a general linear model with repeated measures was implemented with a factor within the groups (fatalism and R_Fatalism) and the Country Group and Project factors.

\subsection{Procedure}

The questionnaires were administered through Caritas Italiana (Caschi Bianchi) operators both directly and by involving local operators, who were responsible for ensuring confidentiality and who answered participants' questions. The questionnaires were filled in individually in writing by each participant in the presence of the operators. Even those who had a low level of education were still able to understand the items perfectly and answer them. Incomplete questionnaires were eliminated. Respondents did not receive any compensation for participating in the study. The volunteers were appropriately trained in the administration by the research team.

The participants first filled out a short form with the socio-demographic variables of interest, such as age, gender, years of study, employment, educational qualifications, etc. They then compiled the Fatalism Scale. After 12 months, and after participating in the intervention project promoted by Caritas, the participants completed the Fatalism Scale (R-Fatalism) again and the General Self Efficacy Scale. The scale used in the retest phase, after 12 months of intervention, was therefore identical to the previous one precisely to detect any changes in the attitude of fatalism. The volunteers were suitably trained for administrating the Scales by the research team, who also supervised the work through email and Skype contacts and with an on-site presence for Argentina and Sri Lanka.

\section{Results}

\subsection{Comparative Analysis}

Preliminary comparisons were made based on a one-way analysis of variance test, concerning the variables of interest of the present study to detect any differences between the four countries. The scores for the fatalism, retest fatalism (R_Fatalism) and GSE scales are shown in Table 1.

Table 1. General self-efficacy, fatalism, R_Fatalism, age, gender, and years of study for the four countries: Argentina $(n=161)$, Bosnia $(n=123)$, Sierra Leone $(n=96)$, and Sri Lanka $(n=130)$.

\begin{tabular}{cccccc}
\hline & Argentina & Bosnia & Sierra Leone & Sri Lanka & \\
\hline Psychological Variables & Mean (SD) & Mean (SD) & Mean (SD) & Mean (SD) & $\mathrm{F}(\mathrm{gl}) p$-value \\
\hline GSE & $33.04(5.43)$ & $28.52(7.23)$ & $31.97(4.33)$ & $31.52(4.99)$ & $15.02_{(3: 489)} p<0.001$ \\
Fatalism & $54.21(20.37)$ & $61.72(23.01)$ & $69.16(16.04)$ & $36.03(8.23)$ & $60.31_{(3: 458)} p<0.001$ \\
R-Fatalism & $37.28(7.96)$ & $37.00(10.74)$ & $39.19(6.38)$ & $41.65(8.61)$ & $7.71_{(3: 489)} p<0.001$ \\
\hline Sociodemographic Variables & Mean (SD) & Mean (SD) & Mean (SD) & Mean (SD) & $\mathrm{F}(\mathrm{gl}) p$-value \\
\hline Age & $34.04(12.41)$ & $33.52(10.15)$ & $36.97(8.50)$ & $34.67(10.64)$ & $2.10_{(3: 498)} p=$ n.s. \\
Gender & $1.87(0.34)$ & $1.54(0.50)$ & $1.30(0.46)$ & $1.80(0.40)$ & $43.87_{(3: 501)} p<0.001$ \\
Years of study & $7.67(3.43)$ & $13.27(2.23)$ & $14.57(2.47)$ & $6.74(3.24)$ & $191.71_{(3: 440)} p<0.001$ \\
\hline
\end{tabular}

\subsection{Correlation Analysis}

A correlational analysis was conducted between the variables of fatalism (fatalism and R_Fatalism) and the scale of self-efficacy (GSE) for the different countries. The results, reported in Table 2, show that the GSE correlates negatively with the fatalism scale in Argentina $(r=-0.188, p=0.011)$ and in Bosnia $(r=-0.244, p=0.005)$ and presents a positive correlation in Sri Lanka $(r=0.530, p<0.001)$, whereas it has no correlation in Sierra Leone $(r=0.51, p=$ n.s.). 
Table 2. P-Correlation between general self-efficacy, and fatalism and R_Fatalism.

\begin{tabular}{ccccc}
\hline & Argentina & Bosnia & Sierra Leone & Sri Lanka \\
\hline Fatalism & $-0.188^{*}$ & $-0.244^{* *}$ & 0.051 & $0.530^{* * *}$ \\
R_Fatalism & $-0.188^{*}$ & $-0.617^{* * *}$ & $-0.224^{*}$ & $-0.540^{* * *}$ \\
\hline \multicolumn{6}{c}{$p<0.05^{* *} p<0.005^{* * *} p<0.001}$.
\end{tabular}

\subsection{Main Analysis}

Hypothesis 1. Social cooperation interventions promote a greater sense of self-efficacy in different populations.

The distribution of the variable welfare project vs. social promotion for the various countries was analyzed and no significant result was found $(\chi 2(3)=0.866, p=$ n.s.). This datum allows to verify whether the project affects the GSE, and therefore, excludes effects linked to the different distributions of the variable in the reference sample.

Considering the different distributions of the variables in the reference sample, gender $(\chi 2(3)$ $=105.07, p<0.001)$ and years of study $(\chi 2(67)=476.91, p<0.001)$ were considered variables of interest to verify the research hypothesis. To validate the research hypothesis, a generalized linear model (ANCOVA) was implemented to verify the effect of the project offered to the participants (welfare project vs. social promotion) in terms of changes in self-efficacy. The model assumed GSE as a dependent variable, country group, and project as fixed factors, and the other variables of interest as covariates (gender, years of study, and R_Fatalism).

The generated model is significant $(\mathrm{F}(10)=15.903, \mathrm{R} 2=0.277, p<0.001)$ and shows significant effects at the country group level $\left(\mathrm{F}(3)=16.427, p<0.001, \eta^{2}=0.106\right)$ and of $\mathrm{R} \_$Fatalism $(\mathrm{F}=79.567$, $p<0.001, \eta^{2}=0.161$ ). The project variable does not assume significance, but in its interaction with the country group variable, it allows to record a significant effect between the subjects (project $\times$ country group $F=3.040, p=0.029, \eta^{2}=0.021$ ). The gender and years of study variables in the generated model do not assume significance. In consideration of the different distribution of the variable years of study in the sample, it was verified whether the education level, or whether having completed at least the basic compulsory education versus having achieved other qualifications, had an impact on the GSE. The results do not reveal any significance (education level: $\mathrm{F}=1.80$, Beta $=-0.064, p=$ n.s.).

A high sense of self-efficacy would also have a predictive effect on the reduction of fatalism (GSE: $\mathrm{F}=90.779$, Beta $=-0.398, p=<0.001, \mathrm{R} 2=159$ ).

Hypothesis 2. The level of fatalism varies over time, following a cooperation intervention and depending on the country of origin.

An analysis of variance was performed for repeated measures to verify the possible differences in the average fatalism scores after a period (12 months) in the four groups after implementation of a welfare project vs. social promotion. In the model, the variables gender and years of study were assumed as covariates. These variables may have an influence on fatalism levels even if it is believed that the most important effect is produced by cooperation intervention: Figures 1 and 2 show the fatalism' effects.

A significant effect of fatalism emerged within the subjects $\left(\mathrm{F}(1,416)=4.51, p<0.034, \eta^{2}=0.011\right)$. Further significant effects are observed within the subjects given by the following interactions: Fatalism $\times \operatorname{GSE}\left(\mathrm{F}(1,416)=4.114, p<0.043, \eta^{2}=0.010\right)$, fatalism $\times$ years of study $(\mathrm{F}(1,416)=23.903, p<0.001$, $\left.\eta^{2}=0.056\right)$, fatalism $\times$ country group $\left(F(1,416)=18.230, p<0.001, \eta^{2}=0.119\right)$, fatalism $\times$ project $\left(\mathrm{F}(3,416)=4.833, p<0.010, \eta^{2}=0.016\right)$, fatalism $\times$ group $\times$ project $(\mathrm{F}(1,416)=8.107, p<0.001$, $\left.\eta^{2}=0.057\right)$. 
Project 1

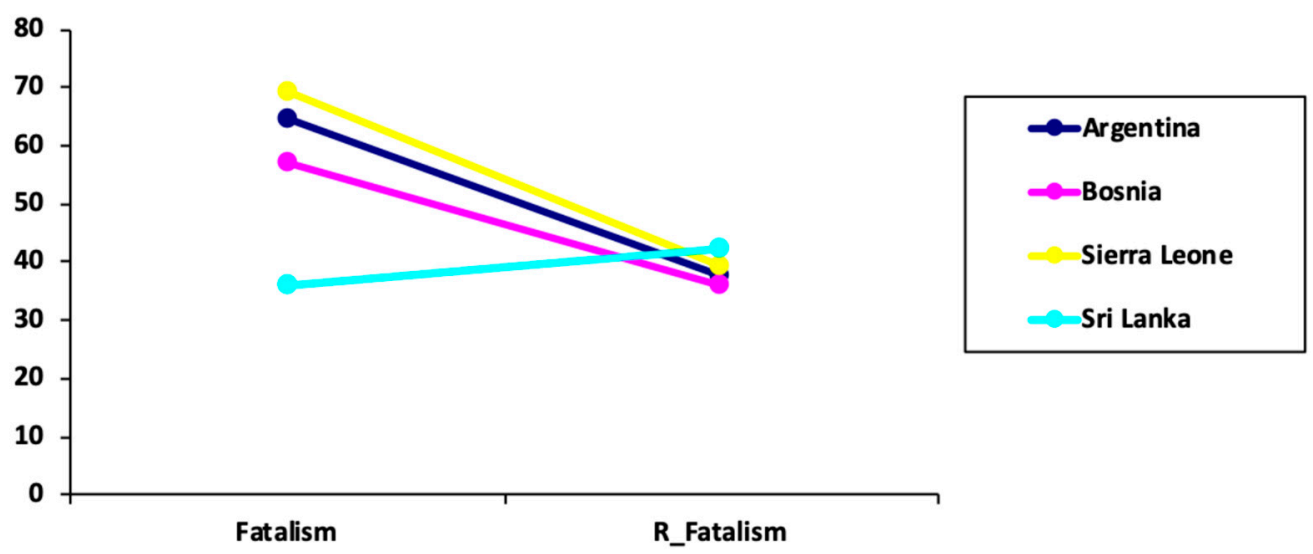

Figure 1. Estimated marginal averages of fatalism in project 1 (welfare).

Project 2

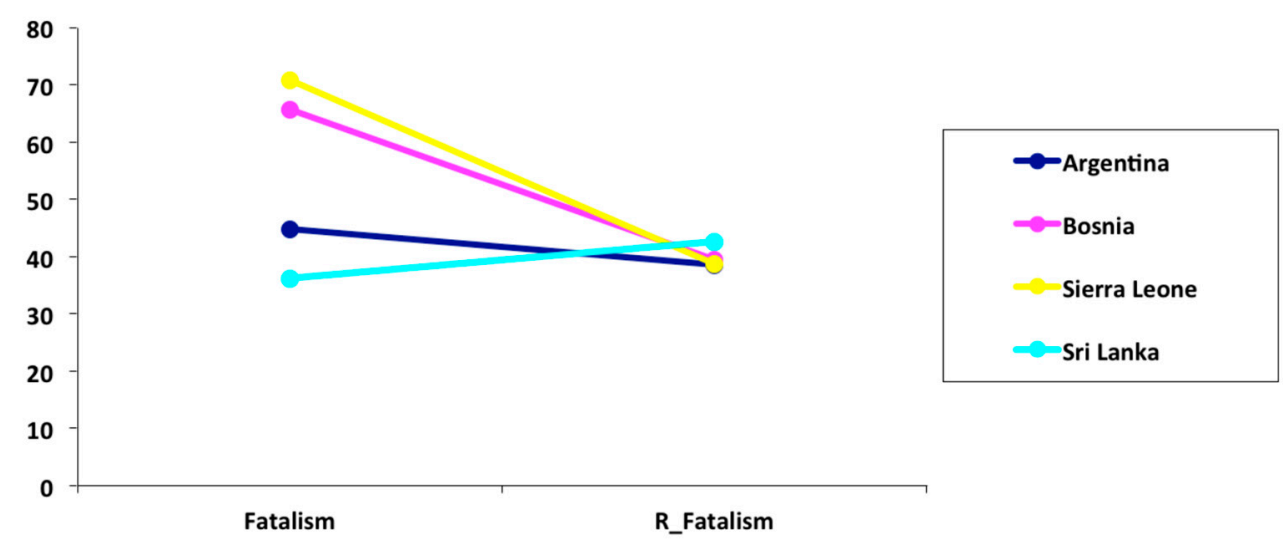

Figure 2. Estimated marginal averages of fatalism in project 2 (social promotion).

At the level of the effects among the subjects, there is a main effect of the country group (test between subjects: $\left.\mathrm{F}(3,416)=25.209, p<0.001, \eta^{2}=0.158\right)$, a main effect of GSE (test between subjects: $\left.F(1,416)=32.822, p<0.001, \eta^{2}=0.075\right)$ and of the variable years of study (test between subjects: $\left.\mathrm{F}(3,416)=8.935, p<0.005, \eta^{2}=0.022\right)$ and a significant interaction between country group $\times$ project (test between subjects $\left.\mathrm{F}(3,416)=13.555, p<0.001, \eta^{2}=0.091\right)$.

Subsequent post-hoc tests, performed with Bonferroni correction, showed significant differences between all countries in average fatalism scores. Table 3 shows the magnitude of the effect recorded between fatalism and R_Fatalism in the two design conditions (welfare vs. social promotion) in each country.

The values of the covariates in the model are as follows: Gender $=1.67$, years of study $=10.31$, GSE $=31.25$. 
Table 3. Comparison of country and project on fatalism.

\begin{tabular}{cccccc}
\hline \multirow{2}{*}{ Country } & Project & $\begin{array}{c}\text { Fatalism Mean } \\
\text { (SD) }\end{array}$ & $\begin{array}{c}\text { R_Fatalism Mean } \\
\text { (SD) }\end{array}$ & \multirow{2}{*}{-Test } & Cohen's d \\
\hline \multirow{2}{*}{ Argentina } & Welfare $(N=74)$ & $63.80(21.73)$ & $37.37(8.08)$ & $8.59^{* * *}$ & 1.61 \\
& Soc. prom. $(N=75)$ & $44.75(13.48)$ & $37.37(6.94)$ & $3.17^{* *}$ & 0.60 \\
Bosnia & Welfare $(N=60)$ & $58.60(20.46)$ & $36.15(8.39)$ & $7.37^{* * *}$ & 1.44 \\
& Soc. prom. $(N=56)$ & $65.32(25.30)$ & $38.79(11.56)$ & $7.27^{* * *}$ & 1.35 \\
Sierra Leone & Welfare $(N=45)$ & $67.84(14.32)$ & $39.69(5.74)$ & $10.28^{* * *}$ & 2.58 \\
& Soc. prom. $(N=50)$ & $70.34(17.51)$ & $38.74(6.92)$ & $9.46^{* * *}$ & 2.37 \\
Sri Lanka & Welfare $(N=42)$ & $36.14(8.41)$ & $42.26(7.59)$ & $-2.55^{*}$ & 0.28 \\
& Soc. prom. $(N=58)$ & $36.17(8.05)$ & $42.69(8.29)$ & $-3.14^{* *}$ & 0.41 \\
\hline \multirow{7}{*}{$* p<0.05^{* *} p<0.005^{* * *} p<0.001}$. & &
\end{tabular}

\section{Discussion}

The results of the present study show a negative correlation in the four countries between the sense of self-efficacy and fatalism, in the retest phase. The sense of self-efficacy tends to contribute to lowering the initial fatalistic attitude and this effect predominates when the participants are involved in sustainability projects that favor better economic assistance conditions and a greater level of social promotion [14]. Self-efficacy and fatalism are transcultural theoretical constructs, but their decrease and association are different in each country because they are strongly linked to cultural aspects. In fact, the interaction between the project and country group variables was significant, indicating that the impact on GSE and fatalism is linked to cultural aspects associated with each country.

In other words, the results of this study confirm that self-efficacy is influenced by cultural aspects, so much so that it does not seem to be as associated with the type of project, economic assistance vs. social promotion, that is offered to the participants, as, instead, to the effect of the interaction of the project $x$ country group. This finding would ultimately tend to indicate how similar projects can lead to different results in different countries, referring to essentially cultural characteristics.

The gender variable does not seem to have significant effects on self-efficacy. However, this lack of effect is considered a limitation of the reference sample that had a significantly higher number of women than men. The different distribution of the gender variable may have affected the final results, which suggests the need to involve more men in future projects. It is also true that the low participation of men could suggest the different attitudes of openness between women and men and differences in their availability to participate in external interventions. These differences are attributable essentially to cultural features.

The level of education does not seem to affect GSE. However, it is necessary to specify that the participants have a low and medium-low level of education because they are from situations of serious social hardship. In collectivist cultures, such as Sierra Leone and Sri Lanka, in particular, the sense of self-efficacy could be linked more to collective, rather than individual, aspects [30]. Therefore, in such contexts, the sense of self-efficacy would not be as linked to the level of education as it would be to cultural aspects. This association could also explain why it is precisely in the countries of Sierra Leone and Sri Lanka that there is less reduction in the attitude of fatalism in the retest phase. These results could suggest that in populations that experience serious economic and social hardships, which are also reflected in the level of education, the sense of self-efficacy could refer to a more concrete dimension of know-how.

Probably, any differences or direct effects related to the education variable could be registered at higher levels (e.g., a degree), which, however, are not present in the reference sample. These considerations suggest that in sustainability projects aimed at particularly disadvantaged populations, for recording a reduction in fatalistic attitudes, in favor of a greater sense of self-efficacy and more active coping strategies, support for the study may also be envisaged. In fact, the results show that the level of education does not have a direct effect on self-efficacy but affects fatalism.

Further, the effect of the sense of self-efficacy in sustainability projects appears to be greater in some countries than in others. These results confirm Scholz et al.'s [39] claim that the concept of 
self-efficacy is less relevant in collectivist cultures than in individualist ones where the role of individual characteristics is more significant. In this sense, we can explain the results obtained for the Sri Lankan participants, with a strong collectivist matrix, where the recorded effect is that of a reinforcement of the initial attitude of fatalism. Although fatalism has reduced in other countries following a cooperation project, Sri Lanka displays a contrasting trend. It is necessary to highlight that Sri Lanka, among the countries considered in the present study, has the lowest level of education and a low socioeconomic level, in general, and in any case, in the considered sample. In fact, according to Ruiu [30], both the level of education and the socioeconomic level affect fatalism. In agreement with other studies [28,29], the presence of a low socioeconomic status is associated with greater fatalism since there would be a perception of the non-supremacy of their disadvantaged condition, despite individual efforts.

In addition to being a collectivist country, Sri Lanka has a strong cultural tendency to adhere to fatalistic, superstitious, and magical concepts. It is possible that planning forms of sustainability in such countries should consider not only support for study, but also different factors for the promotion of autonomy and self-efficacy.

In contrast, in countries such as Sierra Leone and Bosnia, where the level of education is higher (compared with the other countries), there is a greater effect size between fatalism and R_Fatalism. These results seem to be in line with those of Shen et al. [14], who found that a higher level of education is associated with a lower level of fatalism because greater knowledge of information, tools, and personal skills reduces the sense of passive acceptance of one's psychosocial conditions.

As shown in Table 3 and Figures 1 and 2, the reduction of fatalism in the retest phase does not seem to be as linked to the type of project that the participants followed, as to the fact that following or participating in an external project led to a change with respect to their beliefs, skills, and levels of autonomy. The realization of social projects leads the participants to confront themselves with their own cultural aspects, favoring self-efficacy and reduction of fatalistic, magical ideas in favor of greater personal promotion.

The results of this study show that even only the involvement in support interventions can facilitate a process of reduction of merely fatalistic attitudes. Therefore, the possibility of accessing projects aimed at promoting socioeconomic and personal wellbeing urges the overcoming of a passive and ineluctable vision of one's future.

The two project forms considered in this study both influence the reduction of fatalistic attitudes. However, by also considering the cultural variables and the serious difficulties of life the participants experience, the economic aid projects tend to have a greater effect, because these respond to urgent primary needs, refer to a more concrete dimension and, therefore, are probably closer to current needs and to their conception of the ability to overcome external difficulties. Social promotion projects also have a wide effect, and this finding seems to be in line with that of Alvarez et al. [20], who observed that social promotion programs that stimulate autonomy promote greater empowerment strategies.

The results of this research ultimately allow us to detect how the involvement in a social intervention project promotes, in each country, a reduction in individual fatalistic attitudes. Furthermore, projects that facilitate a greater sense of self-efficacy further reduce initial fatalism.

The one-year time period considered, due to the duration and purpose of the various Caritas projects, was a prolonged period of time: Given that fatalism and self-efficacy are processes that require time for their development and their modification it would certainly have been inappropriate to have considered a reduction of times. It would be opportune to consider making a longitudinal comparison in order to evaluate how a longer period of time, as well as a shorter one, might affect the modification processes of fatalism and self-efficacy.

\section{Conclusions}

This study, framed in the perspective of the Psychology of the Sustainability and Sustainable Development [1-3], is focused on the psychosocial variables involved in the description of reactions 
to international cooperation in poor and emerging countries, provides elements to be taken into consideration in designing sustainable international cooperation.

The results obtained should certainly be expanded by detecting data for other countries and with other types of cooperation experiences. Nevertheless, these results allow us to observe how these projects, aimed at improving the economic condition as well as social skills and the sense of community in the disadvantaged populations, allow them to overcome passive beliefs and behaviors such as fatalism, and to increase the ability to feel proactive in improving individuals' and the community's conditions. In fact, it emerges that the help in itself can serve the populations, particularly to meet primary and urgent needs, and that such help has an important meaning in terms of individual and social promotion of the populations themselves. For projects to fully achieve the goals of social promotion and sustainability, it may be useful to bear in mind the cultural context first in the planning process and the relationship with users and, next, the socioeconomic level and the education level, given the correlations highlighted in this study and the possibility of reducing fatalism and promoting self-efficacy.

Author Contributions: Conceptualization, D.P. and M.V.; methodology, M.V. and D.P.; validation, M.V.; formal analysis, M.V.; investigation, D.P., M.V. and V.L.S.; data curation, M.V. and V.L.S.; writing-original draft preparation, D.P., M.V. and V.L.S.; writing - review and editing, S.C. and V.L.S.; visualization, M.V., S.C. and V.L.S.; supervision, S.C.; project administration, D.P. and M.V. All authors have read and agreed to the published version of the manuscript.

Funding: This research received no external funding.

Acknowledgments: We thank the Italian Caritas officials who supported the realization of the research project and the Italian Caritas' volunteers who collaborated in the data collection.

Conflicts of Interest: The authors declare no conflict of interest.

\section{References}

1. Di Fabio, A. The psychology of sustainability and sustainable development for well-being in organizations. Front. Psychol. 2017, 8, 1534. [CrossRef]

2. Di Fabio, A. Positive healthy organizations: Promoting well-being, meaningfulness, and sustainability in organizations. Front. Psychol. 2017, 8, 1938. [CrossRef]

3. Di Fabio, A.; Rosen, M.A. Opening the black box of psychological processes in the science of sustainable development: A new frontier. Eur. J. Sustain. Dev. Res. 2018, 2, 47. [CrossRef]

4. Di Fabio, A.; Kenny, M.E. Intrapreneurial Self-Capital: A Key Resource for Promoting Well-Being in a Shifting Work Landscape. Sustainability 2018, 10, 3035. [CrossRef]

5. Di Fabio, A.; Rosen, M.A. Accounting for Individual Differences in Connectedness to Nature: Personality and Gender Differences. Sustainability 2019, 11, 1693. [CrossRef]

6. Di Fabio, A.; Saklofske, D.H. Positive Relational Management for Sustainable Development: Beyond Personality Traits-The Contribution of Emotional Intelligence. Sustainability 2019, 11, 330. [CrossRef]

7. Di Fabio, A.; Peiró, J.M. Human Capital Sustainability Leadership to promote sustainable development and healthy organizations: A new scale. Sustainability 2018, 10, 2413. [CrossRef]

8. Nussbaum, M.; Sen, A. The Quality of Life; Oxford University Press: Oxford, UK, 1993.

9. Decision on the European Year for Development (2015). Available online: https://eur-lex.europa.eu/eli/dec/ 2014/472/oj (accessed on 3 October 2019).

10. United Nations Objectives for Sustainable Development. Available online: https:/www.un.org/ sustainabledevelopment/inequality/ (accessed on 3 October 2019).

11. International Cooperation by Caritas. Available online: http://www.caritasitaliana.it/home_page/attivita_ /00000968_Cooperazione_Internazionale.html (accessed on 1 August 2019).

12. Powe, B.D. Fatalism among elderly African Americans: Effects on colorectal cancer screening. Cancer Nurs. 1995, 18, 385-392. [CrossRef]

13. Powe, B.D.; Johnson, A. Fatalism as a barrier to cancer screening among African-Americans: Philosophical perspectives. J. Relig. Health 1995, 34, 119-126. [CrossRef] 
14. Shen, L.; Condit, C.M.; Wright, L. The psychometric property and validation of a fatalism scale. Psychol. Health 2009, 24, 597-613. [CrossRef]

15. Bandura, A. Self-Efficacy: Toward a unifying theory of behavioral change. Psychol. Rev. 1977, 84, $191-215$. [CrossRef] [PubMed]

16. Schwarzer, R.; Jerusalem, M. Generalized Self-Efficacy scale. In Measures in Health Psychology: A User's Portfolio. Causal and Control Beliefs; Weinman, J., Wright, S., Johnston, M., Eds.; NFER-Nelson: Windsor, UK, 1995; pp. 35-37.

17. Di Fabio, A.; Tsuda, A. The psychology of harmony and harmonization: Advancing the perspectives for the psychology of sustainability and sustainable development. Sustainability 2018, 10, 4726. [CrossRef]

18. Bandura, A. Social cognitive theory in cultural context. Appl. Psychol. 2002, 51, 269-290. [CrossRef]

19. Neff, J.A.; Hoppe, S.K. Race/ethnicity, acculturation, and psychological distress: Fatalism and religiosity as cultural resources. J. Community Psychol. 1993, 21, 3-20. [CrossRef]

20. Alvarez, K.; van Leeuwen, E.; Montenegro-Montenegro, E.; van Vugt, M. Empowering the poor: A field study of the social psychological consequences of receiving autonomy or dependency aid in Panama. Br. J. Soc. Psychol. 2018, 57, 327-345. [CrossRef]

21. D'Orlando, F.; Ferrante, F.; Ruiu, G. Culturally based beliefs and labor market institutions. J. Socio-Econ. 2011, 40, 150-162. [CrossRef]

22. Perilla, J.L.; Norris, F.H.; Lavizzo, E.A. Ethnicity, culture, and disaster response: Identifying and explaining ethnic differences in PTSD six months after hurricane. J. Soc. Clin. Psychol. 2002, 21, 20-45. [CrossRef]

23. McClure, J.; Sutton, R.M.; Sibley, C.G. Listening to reporters or engineers? How instance-based messages about building design affect earthquake fatalism. J. Appl. Soc. Psychol. 2007, 37, 1956-1973. [CrossRef]

24. Straughan, P.T.; Seow, A. Fatalism reconceptualized: A concept to predict health screening behavior. J. Gend. Cult. Health 1998, 3, 85-100. [CrossRef]

25. Cohen, D.; Nisbett, R.E. Are there differences in fatalism between rural Southerners and Midwesterners? J. Appl. Soc. Psychol. 1998, 28, 2181-2195. [CrossRef]

26. Jacobson, C.K. Denominational and racial and ethnic differences in fatalism. Rev. Relig. Res. 1999, 41, 9-20. [CrossRef]

27. Mechanic, D. Disadvantage, inequality, and social policy. Health Aff. 2002, 21, 48-59. [CrossRef] [PubMed]

28. De Los Monteros, K.E.; Gallo, L.C. Fatalism and cardio-metabolic dysfunction in Mexican-American women. Int. J. Behav. Med. 2013, 20, 487-494. [CrossRef]

29. Freeman, H.P. Cancer in the socioeconomically disadvantaged. CA A Cancer J. Clin. 1989, 39, $266-288$. [CrossRef]

30. Ruiu, G. The origin of fatalistic tendencies: An empirical investigation. Econ. Sociol. 2013, 6, 103-125. [CrossRef] [PubMed]

31. Luszczynska, A.; Gutiérrez-Doña, B.; Schwarzer, R. General self-efficacy in various domains of human functioning: Evidence from five countries. Int. J. Psychol. 2005, 40, 80-89. [CrossRef]

32. Luszczynska, A.; Scholz, U.; Schwarzer, R. The General Self-Efficacy scale: Multicultural validation studies. J. Psychol. 2005, 139, 439-457. [CrossRef]

33. Foster, N.E.; Thomas, E.; Bishop, A.; Dunn, K.M.; Main, C.J. Distinctiveness of psychological obstacles to recovery in low back pain patients in primary care. PAIN@ 2010, 148, 398-406. [CrossRef]

34. Tijou, I.; Yardley, L.; Sedikides, C.; Bizo, L. Understanding adherence to physiotherapy: Findings from an experimental simulation and an observational clinical study. Psychol. Health 2010, 25, 231-247. [CrossRef]

35. Schönfeld, P.; Brailovskaia, J.; Bieda, A.; Zhang, X.C.; Margraf, J. The effects of daily stress on positive and negative mental health: Mediation through self-efficacy. Int. J. Clin. Health Psychol. 2016, 16, 1-10. [CrossRef]

36. Bernard, T.; Dercon, S.; Taffesse, A.S. Beyond fatalism: An empirical exploration of self-efficacy and aspirations failure in Ethiopia. In CSAE Working Paper Series; University of Oxford: Oxford, UK, 2011; Volume 3, pp. 1-24.

37. Taylor, J.; Wilson, J.C. Using our understanding of time to increase self-efficacy towards goal achievement. Heliyon 2019, 5, e02116. [CrossRef] [PubMed]

38. Go, E.; You, K.H. Health-related online information seeking and behavioral outcomes: Fatalism and self-efficacy as mediators. Soc. Behav. Pers. 2018, 46, 871-879. [CrossRef]

39. Scholz, U.; Doña, B.G.; Sud, S.; Schwarzer, R. Is general self-efficacy a universal construct? Psychometric findings from 25 countries. Eur. J. Psychol. Assess. 2002, 18, 242-251. [CrossRef] 
40. International Monetary Fund. Country Composition of WEO Groups. 2015. Available online: http: //www.imf.org/external/pubs/ft/weo/2015/01/weodata/groups.htm\#oem (accessed on 14 November 2019).

41. Tekin, A.K. Parents' motivational beliefs about their involvement in young children's education. Early Child Dev. Care 2011, 181, 1315-1329. [CrossRef] 